\title{
Lesiones urológicas en pacientes sometidas a histerectomía obstétrica por inserción anómala de placenta en un hospital de alta especialidad
}

\author{
Urological lesions in patients undergoing obstetric hysterectomy secondary due to \\ abnormal placental insertion in a high specialty hospital
}

\begin{abstract}
Carlos R. Jiménez-Vieyraㄹ, Alicia Gómez-Suaste², Juan C. Hinojosa-Cruz ${ }^{3}$ y Juan A. García-Bello *
${ }^{1}$ División de Ginecología; '2Servicio de Urología Ginecológica; ${ }^{3}$ Dirección de Educación e Investigación en Salud; ${ }^{4}$ División de Investigación en Salud. Unidad Médica de Alta Especialidad, Hospital de Gineco Obstetricia No. 3 Dr. Víctor Manuel Espinosa de los Reyes Sánchez, Centro Médico Nacional La Raza, Instituto Mexicano del Seguro Social, Ciudad de México, México
\end{abstract}

\begin{abstract}
Resumen
Objetivo: Identificar los factores de riesgo asociados a lesiones urológicas en pacientes con histerectomía por inserción anómala de placenta. Material y métodos: Estudio transversal, retrospectivo, de pacientes sometidas a histerectomía por inserción anómala de placenta de 2017 a 2019. Se estudiaron edad, antecedentes obstétricos, comorbilidad, tipo de inserción placentaria, tipo de histerectomía, sitio de la lesión urinaria, anestesia, tiempo quirúrgico y sangrado. Se realizaron medidas de tendencia central y dispersión, frecuencias y proporciones. Los análisis se realizaron con las pruebas de ji al cuadrado y de Kruskal Wallis con el programa SPSS V.20.0, considerando significativa $p<0.05$. Resultados: Se estudiaron 96 pacientes de $32.6 \pm 6.1$ años. Tuvieron acretismo el $38.5 \%$, incretismo el $19.8 \%$ y percretismo el $18.8 \%$. Se hizo histerectomía total a 78 (81.2\%). Presentaron lesión urinaria 27 (28.1\%): vesical 19, ureteral 6 y ambas 2. La lesión urinaria fue más frecuente en las pacientes que tuvieron más gestas (4 [rango intercuartílico (RIQ): 3-4] vs. 3 [2-4]; $p=0.004$ ), más cesáreas (4 [3-4] vs. 2 [1-3]; $p$ <.001), mayor sangrado (3000 ml [2000-4800] vs. $2000 \mathrm{ml}$ [1200-3000]; $p=0.001$ ) y mayor tiempo quirúrgico (3.75 h [3.5-4.0] vs. 3.0 [2.0-3.5]; $p<0.001)$. Conclusiones: La lesión urinaria fue más frecuente en las pacientes que tuvieron mayor número de gestas, más cesáreas, más sangrado y mayor tiempo quirúrgico.
\end{abstract}

Palabras clave: Histerectomía. Inserción anómala de placenta. Lesión urinaria.

\begin{abstract}
Objective: To describe frequency and identify risk factors for urological lesions in patients undergoing obstetric hysterectomy secondary to abnormal placental insertion in a high specialty hospital. Material and methods: Transversal, retrospective study of patients underwent hysterectomy due to abnormal placental insertion, from 2017 to 2019; age, obstetric antecedents, comorbidities, type of abnormal placental insertion type of hysterectomy, site of urinary lesion, anesthesia, surgery time and bleeding were studied. Analyses were made with ji square and Kruskal Wallis tests with SPSS V20.0 program considering significative $p$ values $p<0.05$. Results: Clinical records of 96 patients with age 32.6 \pm 6.1 years were analyzed. Accretism was present in $38.5 \%$, incretism in $19.8 \%$ and percretism in $18.8 \%$. Total hyster-
\end{abstract}

\footnotetext{
Correspondencia:

*Juan A. García-Bello

Calzada Vallejo, s/n

Col. La Raza

Fecha de recepción: 18-02-2020

C.P. 02990, Azcapotzalco, Ciudad de México, México

E-mail: jagbello67@gmail.com

DOI: 10.24875/CIRU.20000117

Cir Cir. 2020;88(4):473-480

Contents available at PubMed

www.cirugiaycirujanos.com

0009-7411/C 2020 Academia Mexicana de Cirugía. Publicado por Permanyer. Este es un artículo open access bajo la licencia CC BY-NC-ND (http://creativecommons.org/licenses/by-nc-nd/4.0/).
} 
ectomy was accomplished in $78(81.2 \%)$ cases. Urinary lesion was present in $27(28.1 \%)$; vesical in 19 (19.8\%), ureteral in $6(6.5 \%)$ and both in $2(2.0 \%)$. Urinary lesion was significantly more frequent in those patients with more pregnancies (median 4 [interquartile range (IQR): 3-4] vs. 3 [2-4]; $p=0.004$ ), more caesarean sections (4 [3-4] vs. 2 [1-3]; $p<$ 0.001), hemorrhage (3000 mL [2000-4800] vs. $2000 \mathrm{~mL}$ [1200-3000]; $p=0.001)$, and more surgery time (3.75 $h$ [3.5-4.0] vs. 3.0 [2.0-3.5]; $p<0.001)$. Conclusions: Urinary lesions in patients undergoing obstetric hysterectomy secondary to abnormal placental insertion are more frequent with higher number of pregnancies, caesarean sections, hemorrhage and surgery time.

Key words: Hysterectomy. Abnormal placental insertion. Urological lesion.

\section{Introducción}

La placentación anómala se refiere a la inserción anormal de parte o de toda la placenta, con ausencia total o parcial de la decidua basal y anormalidad de la decidua verdadera con penetración de las vellosidades coriales al miometrio'.

Existen tres variedades de adherencia anormal de la placenta2: la placenta acreta es la adherencia anormal de una parte o de la totalidad de la placenta a la pared uterina sin que las vellosidades coriales penetren el miometrio; la placenta increta es aquella en la que las vellosidades coriales penetran el miometrio; y la placenta percreta se presenta cuando hay penetración de los elementos coriales hasta sobrepasar la serosa del útero, pudiendo alcanzar órganos vecinos. Por su extensión puede ser focal, cuando solo involucra pequeñas áreas de la placenta; parcial, cuando uno o más cotiledones se involucran en el proceso; o total, cuando la superficie completa de la placenta está anormalmente adherida ${ }^{3}$. El término general de placenta acreta incluye todas las anomalías de invasión placentaria, independientemente de la profundidad de la invasión ${ }^{4}$. La placenta percreta es la forma más grave y se define como la penetración en su totalidad del espesor del miometrio por las vellosidades que perforan el útero y el peritoneo visceral ${ }^{5}$. El riesgo hemorrágico está ligado a la rotura de los vasos uterinos por las vellosidades y sus enzimas proteolíticas, y a la imposibilidad de despegamiento de la placenta en el momento del alumbramiento ${ }^{6}$. Cuando la placenta percreta se inserta sobre el segmento inferior (70\% de los casos), las complicaciones propias de una inserción baja aumentan, pero si estas dos eventualidades ocurren sobre la cicatriz de una o varias cesáreas, se reúnen las condiciones para que las vellosidades se rompan y pasen a través de una cicatriz, en ocasiones dehiscente, produciéndose una rotura uterina silenciosa, en cualquier fase de la gestación o en el parto7. Una vez producida la rotura uterina, las vellosidades placentarias pueden seguir su progresión e invadir las estructuras pélvicas vecinas, como la vejiga urinaria, y afectar al trígono vesical y los dos orificios ureterales, el espacio de Retzius, el epiplón, el mesenterio y los vasos ilíacos ${ }^{5}$.

Los síntomas principales son sangrado y signos urológicos, que pueden comprender disuria o polaquiuria sin infección urinaria, pero los más significativos son las hematurias que pueden aparecer desde la semana 19. Al principio la mayoría son microscópicas $^{8}$, son siempre recidivantes y pueden ser preoperatorias, intraoperatorias 0 aparecer más tarde.

Los principales factores de riesgo para una implantación anómala de placenta $\operatorname{son}^{9}$ la edad, la multiparidad, el antecedente de cesárea con placenta previa, la placenta previa con antecedente de tres o más cesáreas, antecedente de legrado uterino, antecedente de extracción manual placentaria, antecedente de retención placentaria y antecedente de infección intraamniótica. La placenta previa sola, sin cirugía uterina previa, se asocia con un riesgo de acretismo del $1-4 \%{ }^{10}$. En un estudio previo en el Instituto Nacional de Perinatología, de 210 casos de placenta previa estudiados, en 37 (17.6\%) se presentó acretismo placentario; de ellos, en 26 (70.2\%) había antecedente de cesárea. Los autores demostraron que, a mayor número de cesáreas, la frecuencia de acretismo fue mayor: el $9.4 \%$ en las pacientes que no tenían cesáreas previas, el $21.1 \%$ en las que tenían una y el $50 \%$ en las que habían tenido dos o más cesáreas. El grupo de edad con la frecuencia más alta de acretismo (26\%) fue el de 35-39 años ${ }^{11}$.

La placenta acreta se presenta en 1 de cada 2500 nacimientos, con una incidencia que varía entre el 0,001 y el $0,9 \%$ de los partos ${ }^{12}$. El diagnóstico prenatal y el conocimiento del alcance de la invasión placentaria son fundamentales para optimizar los resultados de las pacientes con placenta acreta ${ }^{2}$. Las consecuencias de la placenta acreta pueden ser graves, con tasas de mortalidad materna de alrededor del $7 \%{ }^{13}$. La vía de resolución es por medio de operación 
cesárea, de preferencia programada y con un enfoque multidisciplinario, que incluya al servicio de uro-ginecología, así como un cirujano vascular. Se prefiere el empleo de inductores de la madurez pulmonar y, una vez documentada esta por amniocentesis a las 36-37 semanas, se interrumpe la gestación por cesárea. La resolución puede realizarse antes si existiese una urgencia, como es el sangrado incoercible, o si la paciente inicia el trabajo de parto'.

El principal tratamiento es la histerectomía obstétrica total, debido a su frecuente asociación con placenta previa y cicatrices de cesáreas previas. Este procedimiento es un desafío a las habilidades quirúrgicas del médico obstetra ${ }^{3}$. La histerectomía puede conducir a complicaciones por lesión de órganos adyacentes y a comorbilidad grave ${ }^{14}$.

El manejo de la placenta acreta puede acompañarse de lesiones en el intestino o de rotura uterina ${ }^{15}$. La lesión del tracto urinario durante la histerectomía por cesárea para la placenta invasiva es tan alta como del $29 \%$. En el contexto de hematuria persistente, normovolemia, salida de orina por la vagina, oliguria y anuria debe considerarse una lesión urológica no reconocida ${ }^{16}$. Las lesiones vesicales ocurren en el $6-29 \%$ de los casos y las lesiones ureterales en el $2-6 \%{ }^{17}$. En un estudio previo, esta morbilidad materna fue tan alta que en 39 de 54 casos reportados de invasión vesical hubo lesión urológica, con lesión vesical en el $26 \%$, fístula urinaria en el $13 \%$, hematuria macroscópica en el $9 \%$, lesión ureteral en el $6 \%$, disminución de la capacitancia vesical en el $4 \%$ y cistectomía en el $44 \%$; en cuanto a la mortalidad, se produjeron $3(5,6 \%)$ muertes maternas y $14(25,9 \%)$ muertes fetales.

En el caso del tratamiento radical de la placenta percreta, la colocación de un catéter doble J por sospecha no parece alterar el riesgo de complicaciones ${ }^{18}$.

Las lesiones en la vía urinaria pueden ser diagnosticadas de forma intraoperatoria, como la laceración en la vejiga y el uréter, o posoperatoria, como la fístula vesicovaginal, la fístula ureterovaginal y la estenosis uretral ${ }^{19}$.

No encontramos en la literatura ningún reporte previo de los factores de riesgo asociados a estas complicaciones en este grupo específico de pacientes. Tales datos son necesarios para conocer la magnitud del problema, ya que la inserción anómala de la placenta es frecuente. Identificar de manera temprana los factores de riesgo asociados a la lesión urinaria nos permitirá anticipar las medidas necesarias, tanto para el diagnóstico como para el tratamiento de la inserción anómala de la placenta, con el fin de evitar, en la medida de lo posible, la presentación de estas complicaciones.

El objetivo de este estudio fue identificar la frecuencia de lesión urinaria y los factores de riesgo asociados a ella en pacientes sometidas a histerectomía obstétrica por inserción anómala de placenta en un hospital de alta especialidad.

\section{Método}

Previa autorización por el Comité de Ética en Investigación y del Comité Local de Investigación del Hospital, se llevó a cabo un estudio observacional, retrospectivo, transversal y analítico, en el que se revisaron los registros clínicos de pacientes sometidas a histerectomía por inserción anómala de placenta en la Unidad Médica de Alta Especialidad Hospital de Gineco Obstetricia No. 3 Dr. Víctor Manuel Espinosa de los Reyes Sánchez, del Centro Médico Nacional La Raza, hospital de tercer nivel en el Instituto Mexicano del Seguro Social, de enero de 2017 a enero de 2019. Se registraron la paridad, la edad de la paciente, el antecedente de cesáreas previas, el tipo de anomalía de inserción placentaria, el tipo de histerectomía (total o subtotal), el tiempo quirúrgico, el sangrado estimado y la presencia o no de lesión de tracto urinario, y si fue el caso, el sitio de esta. Para la estadística descriptiva se utilizaron frecuencias, proporciones, medidas de tendencia central y dispersión, y para buscar la asociación entre los distintos factores de riesgo y la presentación de lesión de la vía urinaria se utilizaron las pruebas t de Student o U de Mann-Whitney, ji al cuadrado o exacta de Fisher, y de Kruskal-Wallis, con el paquete estadístico SPSS versión 22.0. Se consideraron significativos los valores de $p<0.05$.

El estudio obtuvo el número de registro por los Comités de Ética en Investigación y de Investigación en Salud, y se apegó a los principios éticos de la Declaración de Helsinki y al Reglamento de la Ley General de Salud en materia de investigación y normatividad que de ellas se desprende. Se trata de un estudio sin riesgo, en una población vulnerable (la mujer embarazada), con un balance riesgo-beneficio adecuado. En todo momento se preserva la confidencialidad de las participantes, pues las bases de datos contienen solo números de folio y la información que pudiera ayudar a la identificación de las participantes se mantiene codificada aparte y en posesión del investigador principal (C.R. Jiménez Vieyra). 


\section{Resultados}

Se estudiaron los registros clínicos de 96 pacientes con una edad de $32.6 \pm 6.1$ años (rango: 18-46). La mediana de gestas fue de 3 , con intervalo intercuartílico [RIQ] de 2-4; la de cesáreas fue de 2 [1-3]. Tuvieron diabetes mellitus 7 (7.3\%) pacientes, e hipertensión arterial $7(7.3 \%)$ pacientes. Otras enfermedades menos frecuentes fueron lupus eritematoso sistémico, hipotiroidismo, prolapso de válvula mitral, epilepsia, trombosis venosa profunda, asma y artritis reumatoide (una paciente en cada caso). Presentaron acretismo placentario 37 (38.5\%) pacientes, 19 (19.8\%) placenta increta, 18 (18.8\%) percretismo placentario y 12 (12.5\%) placenta previa documentada por reporte ultrasonográfico; en 10 (10.4\%) pacientes no fue posible documentar el tipo exacto de inserción anómala por estudio histopatológico. La mediana de sangrado fue de $2000 \mathrm{ml}$ [1300-3425] (rango: 500-7000) y la media del tiempo quirúrgico fue de 3 horas [2.5-3.5].

A 78 de 96 pacientes (81.3\%) se les realizó histerectomía total y a 18 de 96 (18.8\%) subtotal. Se realizó histerectomía total a 32 de las 37 pacientes con acretismo (86.5\%), a 14 de las 19 con placenta increta (73.7\%), a 16 de las 18 con percretismo (88.9\%), a 10 de las 12 con placenta previa (83.3\%) y a 6 de las 10 en las que no se documentó histológicamente el tipo de inserción anómala (60\%).

En 27 (28.1\%) pacientes se presentó lesión del tracto urinario (Tabla 1). Respecto al tipo de inserción anómala que tenían, 12 (44.5\%) tuvieron placenta percreta, $6(22.2 \%)$ placenta acreta, $6(22.2 \%)$ placenta increta, $2(7.4 \%)$ placenta previa y en $1(3.8 \%)$ no se identificó histológicamente el tipo. En 21 de 27 pacientes $(77.8 \%)$ fue necesaria la histerectomía total y en 6 de 27 (22.2\%) se realizó histerectomía subtotal. Fue posible realizar una histerectomía subtotal a tres pacientes con placenta percreta, a una con placenta acreta, a una con placenta increta y a una con placenta previa.

Según el sitio de la lesión, en 19 de 27 pacientes (70.4\%) la lesión fue vesical, en 6 de 27 (22.2\%) fue ureteral y en 2 de 27 (7.4\%) fue tanto de vejiga como de uréter. De las 19 lesiones vesicales, nueve fueron invasión, cuatro fístulas, cinco sección y una ligadura. De las seis lesiones uretrales, cinco fueron ligaduras de uréter y una sección. De las dos pacientes que tuvieron lesión vesical y uretral, ambas presentaron fístula, una con invasión y otra con sección. De las 19 lesiones vesicales, en 15 el diagnóstico se hizo intraoperatorio y en cuatro en el periodo posoperatorio; de las seis pacientes con lesión ureteral, en tres el diagnóstico fue transoperatorio y en tres fue posoperatorio; y para las pacientes con lesión en ambos sitios, una fue diagnosticada en el transoperatorio y otra en el posoperatorio. De las 19 lesiones vesicales, en 11 el diagnóstico se hizo por visualización directa, en cuatro usando azul de metileno y en cuatro por cistoscopía. Para el diagnóstico de las seis lesiones uretrales, tres fueron por visualización directa, dos por ureterocistoscopía y una por uro tomografía y urografía excretora. Una paciente con lesión en la vejiga y el uréter fue diagnosticada mediante ureterocistoscopía, y la otra por visualización directa, urotomografía y urografía excretora.

En lo que respecta al abordaje de la lesión a la vía urinaria, solo en 9 de los 27 casos fue reparada por el ginecólogo; en los 18 restantes, lo fue por el especialista en urología. El tipo de anestesia más utilizada fue la regional, en $61(63.5 \%)$ pacientes, seguida de la general en 35 (36.5\%).

Al comparar a las pacientes según el tipo de implantación anómala de la placenta, en aquellas con placenta previa el número de cesáreas previas y el sangrado fueron significativamente menores, mientras que el sangrado fue significativamente mayor en las pacientes con placenta percreta. No hubo diferencias significativas en cuanto a la edad, el número de gestas ni el tiempo quirúrgico (Tabla 2).

En el análisis para identificar los factores de riesgo asociados a las lesiones de la vía urinaria, las pacientes que tuvieron lesión de la vía urinaria tuvieron un número significativamente mayor de gestas (mediana 4 [3-4] vs. 3 [2-4]; $p=0.004$ ), más cesáreas (mediana 4 [3-4] vs. 3 [1-3]; $p=0.004)$, mayor frecuencia de percretismo placentario (70.6 vs $29.4 \% ; p=0.001$ ), mayor tiempo quirúrgico (mediana $3.7 \mathrm{~h}$ [3.5-4.0] vs. $3.0 \mathrm{~h}$ [2.0-3.5]; $p<0.001$ ) y más sangrado transoperatorio (mediana $3000 \mathrm{ml}$ [2000-4800] vs. $2000 \mathrm{ml}$ [1200-3000]; $p=0.001$ ), y en ellas fue más frecuente el uso de anestesia general (57.1 vs. $42.9 \%$; $p<0.001$ ) que en las pacientes que no tuvieron lesión (Tabla 3).

\section{Discusión}

El Hospital de Gineco Obstetricia No. 3 del Centro Médico Nacional La Raza es un hospital de referencia de pacientes con inserción anómala de placenta. En promedio, en este centro hospitalario se atienden al año más de 128 mujeres con esta patología asociada al embarazo, y puede ser una condición que amerite incluso la realización de histerectomía obstétrica. 

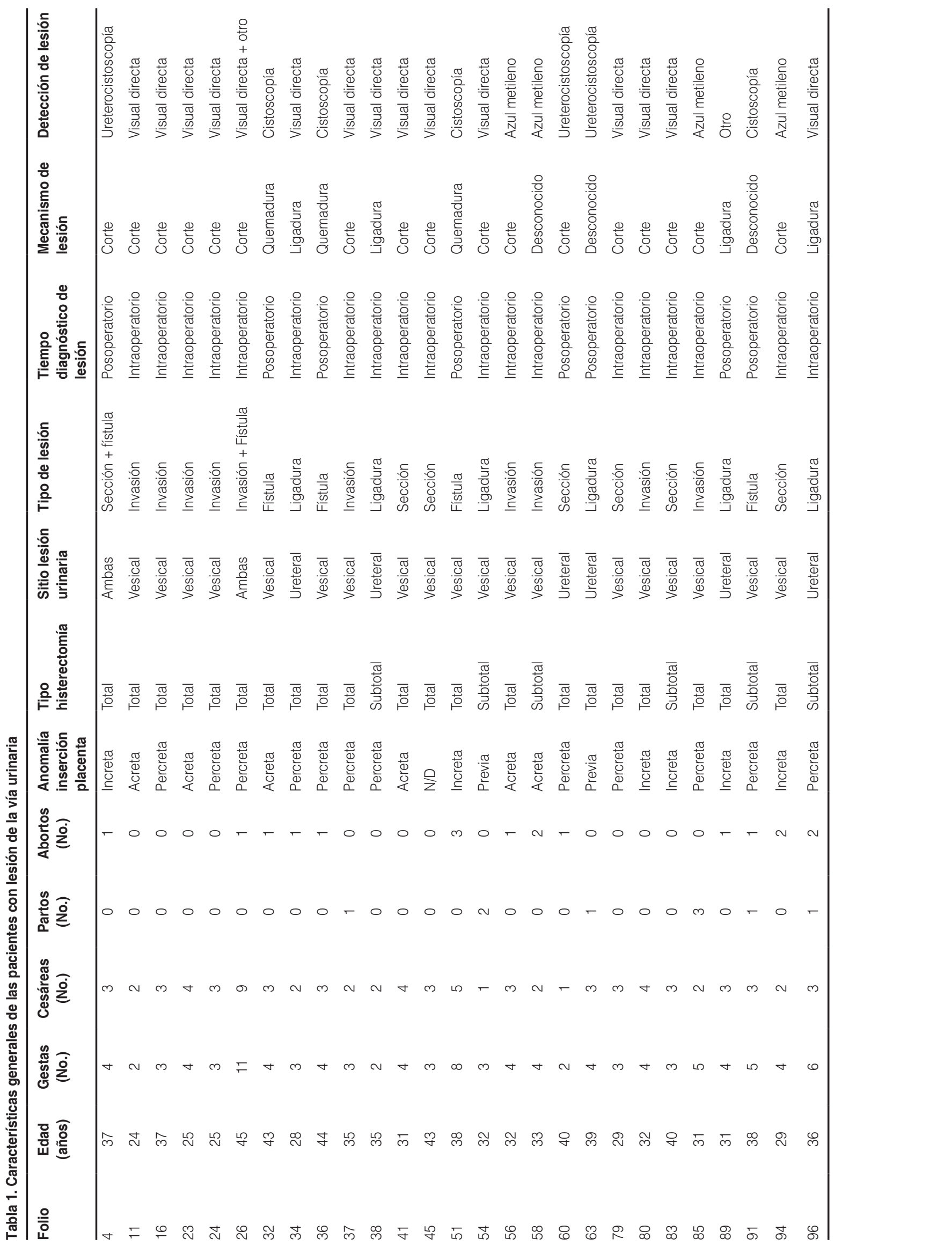
Cirugía y Cirujanos. 2020;88(4)

Tabla 2. Características según el tipo de implantación anómala

\begin{tabular}{|c|c|c|c|c|c|c|}
\hline \multirow[t]{2}{*}{ Variable } & \multirow[t]{2}{*}{ Total $(n=96)$} & \multicolumn{4}{|c|}{ Tipo de inserción anómala } & \multirow[t]{2}{*}{$\mathrm{p}$} \\
\hline & & Acreta $(n=35)$ & Increta $(n=16)$ & Percreta $(n=17)$ & Previa $(n=11)$ & \\
\hline Edad (años) & 32 [28-38] & 31 [28-35] & 35 [31-40] & 36 [30-40] & 32 [27-39] & 0.100 \\
\hline Gestas & $3[2-4]$ & $3[2-4]$ & $4[3-4]$ & $3[2-5]$ & 2 [2-3] & 0.059 \\
\hline Cesáreas & $2[1-3]$ & $2[2-3]$ & $3[1-3]$ & $3[2-3]$ & $1[1-2]$ & $0.029^{*}$ \\
\hline Sangrado (ml) & 2000 [1300-3425] & 2000 [1200-3000] & 2900 [1800-3950] & 3500 [1900-4900] & $1500[1200-2500]$ & $0.016^{*}$ \\
\hline Tiempo quirúrgico (h) & $3.0[2.5-3.5]$ & $3.0[2.5-3.5]$ & $3.5[3.0-4.0]$ & $3.5[2.0-4.0]$ & $3.0[2.5-3.7]$ & 0.564 \\
\hline
\end{tabular}

*Prueba de Kruskal Wallis.

Se reportan medianas [intervalo intercuartílico].

Tabla 3. Características según la presencia de lesión de la vía urinaria

\begin{tabular}{|c|c|c|c|c|c|}
\hline \multirow[t]{2}{*}{ Variable } & & \multirow[t]{2}{*}{ Total $(n=96)$} & \multicolumn{2}{|c|}{ Lesión de la vía urinaria } & \multirow[t]{2}{*}{$p$} \\
\hline & & & No $(n=69)$ & Sí (n = 27) & \\
\hline Edad (años) & & $32(28-38)$ & $32(28-37)$ & $35(31-39)$ & 0.080 \\
\hline Gestas & & $3(2-4)$ & $3(2-4)$ & $4(3-4)$ & $0.004^{*}$ \\
\hline Cesáreas & & $2(1-3)$ & $3(1-3)$ & $4(3-4)$ & $<0.001^{*}$ \\
\hline \multirow{4}{*}{$\begin{array}{l}\text { Anomalía de } \\
\text { inserción, N (\%) }\end{array}$} & Previa & $11(100)$ & $9(81.8)$ & $2(18.2)$ & \multirow[t]{4}{*}{$0.001^{\dagger}$} \\
\hline & Acreta & $35(100)$ & $29(82.9)$ & $6(17.1)$ & \\
\hline & Increta & $16(100)$ & $10(62.5)$ & $6(37.5)$ & \\
\hline & Percreta & $17(100)$ & $5(29.4)$ & $12(70.6)$ & \\
\hline \multirow[t]{2}{*}{ Tipo de histerectomía, N (\%) } & Total & $78(100)$ & $57(73.1)$ & $21(26.9)$ & \multirow[t]{2}{*}{0.586} \\
\hline & Subtotal & $18(100)$ & $12(66.7)$ & $6(33.3)$ & \\
\hline \multirow[t]{2}{*}{ Tipo de anestesia, N (\%) } & Regional & $61(100)$ & $54(88.5)$ & $7(11.5)$ & \multirow[t]{2}{*}{$<0.001^{\dagger}$} \\
\hline & General & $35(100)$ & $15(42.9)$ & $20(57.1)$ & \\
\hline Sangrado (ml) & & 2000 (1300-3425) & 2000 (1200-3000) & $3000(2000-4800)$ & $0.001^{*}$ \\
\hline Tiempo quirúrgico (h) & & $3.0(2.5-3.5)$ & $3.0(2.0-3.5)$ & $3.7(3.5-4.0)$ & $<0.001^{*}$ \\
\hline
\end{tabular}

Dicho procedimiento es complejo por tratarse de un padecimiento asociado a hemorragia obstétrica, la cual condiciona una alta tasa de morbimortalidad materna y fetal, y puede afectar el futuro reproductivo de quien la padece ${ }^{2}$.

En nuestro estudio se presentaron 63 casos en mujeres mayores de 30 años, lo que coincide con los datos reportados con anterioridad ${ }^{9}$. El $72.9 \%$ de las pacientes no presentaban alguna enfermedad que elevará el riesgo de implantación anómala de la placenta, y no identificamos asociación significativa entre la presencia de lesión de la vía urinaria y la comorbilidad de las pacientes, que en su mayoría se trataba de padecimientos crónicos como hipertensión y diabetes mellitus.

Con respecto al antecedente de operación cesárea al realizar la histerectomía obstétrica, se observó que el $33.3 \%$ de las pacientes fueron secundigestas con una cesárea previa, una paciente fue trigesta con dos cesáreas previas, y el número mayor de cesáreas correspondió a una paciente en su undécima gestación con nueve cesáreas previas; en total, el $69.8 \%$ de las pacientes tuvieron cesáreas previas, 
lo que coincide con lo publicado por $\mathrm{Wu}$, et al. ${ }^{9}$, quienes reportan un porcentaje de cesárea previa del $67 \%$. Por otra parte, se presentaron tres casos de histerectomía obstétrica en primigestas $(2,8 \%)$; en dos de ellas, la histerectomía se realizó durante su primera cesárea, y en otra paciente fue posparto, corroborando por histopatología el diagnóstico de acretismo placentario, a pesar de no contar, teóricamente, con factores de riesgo asociados, lo que también coincide con la literatura previa, en la que se ha encontrado riesgo de placentación anómala incluso sin antecedentes quirúrgicos en el $1-4 \%$ de las pacientes ${ }^{10}$.

El tipo de histerectomía realizada con mayor frecuencia fue la histerectomía total $(81.3 \%$ de los casos). A pesar de que la literatura señala que el tipo de histerectomía debe ser individualizado, hay preferencia por realizar la histerectomía subtotal debido a que se considera que puede reducir el tiempo quirúrgico ${ }^{20}$. Sin embargo, en este trabajo se documentó un mayor porcentaje de lesión a la vía urinaria con la histerectomía subtotal (33\%), lo que elevó el tiempo quirúrgico para su reparación.

De las 27 pacientes que presentaron lesión a la vía urinaria, el sitio más frecuente fue la vejiga, en 19 de las 27 pacientes $(70.4 \%)$, seguida del uréter en 6 de las $27(22.2 \%)$. Lo anterior coincide con la literatura, que reporta una frecuencia de lesión vesical que va del 6 al 29\% y de lesión ureteral del 2 al 6\% ${ }^{17}$. Según reportes previos, durante la histerectomía secundaria a cesárea por placenta invasiva la frecuencia de lesión del tracto urinario es tan alta como del $29 \%{ }^{18}$; sin embrago, en nuestro estudio identificamos 11 casos con lesión a la vía urinaria, de los cuales 7 (63.3\%) tuvieron lesión vesical, lo que supone un porcentaje dos veces mayor que lo publicado. Creemos que esto se debe a que nuestro estudio incluyó grados mayores de invasión y una mayor frecuencia de factores de riesgo asociados a esta invasión, lo cual hizo que nuestra casuística se incrementará.

Afortunadamente, la detección de la lesión se realizó de forma intraoperatoria en el $70 \%$ de los casos, lo que mejoró el pronóstico de las pacientes y disminuyó el riesgo de complicaciones posquirúrgicas, como también fue reportado por Washecka y Behling ${ }^{18}$, debido a la correcta y oportuna reparación de la lesión.

La reparación corrió a cargo del servicio de urología en el $66.3 \%$ de los casos, y en el resto la realizó el servicio de ginecología, con personal adiestrado para la reparación, lo que destaca la importancia de la atención por un equipo multidisciplinario ${ }^{13}$.
En ningún caso fue necesaria la cateterización de uréteres con la finalidad de prevenir la lesión a pesar de la sospecha de percretismo placentario; de todos modos, estudios previos señalan que dicho procedimiento no suele alterar el riesgo de complicaciones ${ }^{18}$.

El hallazgo en nuestro estudio de que un mayor sangrado se asocia a una mayor probabilidad de lesión de la vía urinaria confirma lo reportado previamente por Alcocer, et al. ${ }^{21}$, quienes también lo identificaron entre los factores de riesgo en mujeres con lesión urinaria durante la operación cesárea ${ }^{21}$.

Respecto a la búsqueda de los factores de riesgo asociados a la presencia de lesión del tracto urinario, estudios previos se han centrado en grupos pequeños de pacientes ginecológicas ${ }^{22,23}$ o en pacientes oncológicas $^{24}$. Después de nuestra búsqueda en la literatura, hasta donde sabemos, este es el primer estudio en pacientes con lesión urinaria secundaria a histerectomía obstétrica por inserción anómala de placenta.

\section{Conclusiones}

Los factores de riesgo para lesión de la vía urinaria en pacientes que tuvieron histerectomía obstétrica por inserción anómala de placenta en nuestro estudio fueron el antecedente de múltiples gestas, cesáreas previas, grados mayores de inserción anómala, mayor tiempo quirúrgico y mayor sangrado durante la cirugía.

La inserción anómala de placenta es una condición obstétrica que pone en peligro la vida de la mujer embarazada. Actualmente hay un incremento en la realización de cesáreas, legrados y cirugías uterinas. El antecedente de alguna de estas cirugías es un factor de riesgo importante para la presencia de alteraciones de la placentación, para la realización de cesárea y para la necesidad de histerectomía obstétrica.

La lesión de la vía urinaria es una complicación que puede llevar a estancias prolongadas, y comporta mayor riesgo de otras complicaciones como infecciones o eventos trombóticos, y en consecuencia, un mayor gasto para las pacientes, para sus familias y para los servicios de salud.

Es necesario que el médico urólogo y el especialista en urología ginecológica estén capacitados en el manejo preoperatorio, transoperatorio y posoperatorio de esta complicación.

\section{Conflicto de intereses}

Los autores manifiestan que no tienen ningún conflicto de intereses respecto de este manuscrito. 


\section{Responsabilidades éticas}

Protección de personas y animales. Los autores declaran que para esta investigación no se han realizado experimentos en seres humanos ni en animales.

Confidencialidad de los datos. Los autores declaran que han seguido los protocolos de su centro de trabajo sobre la publicación de datos de pacientes.

Derecho a la privacidad y consentimiento informado. Los autores declaran que en este artículo no aparecen datos de pacientes. Este estudio cuenta con aprobación por el Comité de Ética en Investigación en Salud y del Comité Local de Investigación en Salud R-2019-3504-010.

\section{Bibliografía}

1. Instituto Nacional de Perinatología. Normas y procedimientos en Ginecología y Obstetricia del Instituto Nacional de Perinatología de México. Edición 2003. Marketing y Publicidad de México; 2003. p. 129-32.

2. Wortman AC, Alexander JM. Placenta accreta, increta, and percreta. Obstet Gynecol Clin North Am. 2013;40:137-54.

3. Dueñas GO, Rico OH, Rodríguez BM. Actualidad en el diagnóstico y manejo del acretismo placentario. Rev Chil Obstet Ginecol. 2007;72:266-71.

4. Secretaría de Salud. Guía de práctica clínica para el diagnóstico y manejo de las anomalías de inserción placentaria y vasos sanguíneos fetales. México: Secretaria de Salud; 2013.

5. Héquet D, Ricbourg A, Sebbag D, Rossignol M, Lubrano S, Barranger E. Placenta accreta: dépistage, prise en charge et complications. Gynécologie Obstétrique \& Fertilité. 2013:41:31-7.

6. Gil FR, Barrero H, Pastor F, Gil GF. Placenta percreta sobre cicatriz de cesárea con invasión vesical. Prog Obstet Ginecol. 2001;44:180-6.

7. Klotz $\mathrm{P}$, Khalaff $\mathrm{H}$. Placenta percreta invading the bladder: report of 2 cases. J Urol. 1998:141:938-9.

8. Mégier P, Gorin V, Desroches A. Placentas bas insérés échographiquement au 30 trimestre de la grossesse: recherche de signes de placenta accreta/percreta et de vaisseaux praevia. Étude prospective en échographie Doppler couleur et pulsé de 45 cas. J Gynecol Obstet Biol Reprod. 1999;28:239-44

9. Wu S, Kocherginsky M, Hibbard JU. Abnormal placentation: twenty-year analysis. Am J Obstet Gynecol. 2005;192:1458-61.

10. Silver RM, Landon MB, Rouse DJ, Leveno KJ, Spong CY, Thom EA, et al. Maternal morbidity associated with multiple repeat cesarean deliveries. Obstet Gynecol. 2006;107:1226-32.

11. Lira J, Ibarbuengoitia F, Argueta M, Karchmer S. Placenta previa/acreta y cesárea previa. Experiencia de cinco años en el Instituto Nacional de Perinatología. Ginec Obst Mex. 2005;638:337-40.

12. Gielchinsky Y, Rojansky N, Fasouliotis SJ, Ezra Y. Placenta accreta: summary of 10 years: a survey of 310 cases. Placenta. 2002;23:210-4.

13. Eller AG, Bennet MA, Sharshiner M, Masheter C, Soisson AP, Dodson $\mathrm{M}$, et al. Maternal morbidity in cases of placenta accreta managed by a multidisciplinary care team compared with standard obstetric care. Obstet Gynecol. 2011;117:331-7.

14. Usta IM, Hobeika EM, Musa AA, Gabriel GE. Placenta previa-accreta: risk factors and complications. Am J Obstet Gynecol. 2005;193:1045-9.

15. Wilches-Llanos A, Palazuelos-Jiménez G, Trujillo-Calderón S, Romero-Enciso J. Diagnóstico prenatal de acretismo placentario: hallazgos y utilidad del ultrasonido y la resonancia magnética: reporte de casos en el Hospital Universitario de la Fundación Santa Fe de Bogotá (Colombia). Rev Colomb Obstet Ginecol. 2014;65:346-53.

16. Tam KB, Dozier J, Martin JN Jr. Approaches to reduce urinary tract injury during management of placenta accreta, increta, and percreta: a systematic review. J Matern Fetal Neonatal Med. 2012;25:329-34.

17. Wright JD, Bonanno C, Shah M, Gaddipati S, Devine P. Peripartum hysterectomy. Obstet Gynecol. 2010;116:429-34

18. Washecka R, Behling A. Urologic complications of placenta percreta invading the urinary bladder: a case report and review of the literature. Hawaii Med J. 2002;61:66-9.

19. Bai SW, Huh EH, Jung da J, Park JH, Rha KH, Kim SK, et al. Urinary tract injuries during pelvic surgery: incidence rates and predisposing factors. Int Urogynecol J Pelvic Floor Dysfunct. 2006;17:360-4.

20. Kastner ES, Figueroa R, Garry D, Maulik D. Emergency peripartum hysterectomy: experience at a community teaching hospital. Obstet Gynecol. 2002;99:971-5

21. Alcocer UJ, Bonilla MM, Gorbea CV, Velázquez VB. Factores de riesgo asociados a lesiones vesicales en cesárea. Acta Urol Esp. 2009;33:806-10.

22. Ridha F, Latifa M, Ines R, Maamar H, Salem B. Ureteral injuries complicating gynecologic surgery. Pan Afr Med J. 2018:30:1-10.

23. Messaoudi F, Ben Jemaa S, Yazidi M, El Housseini S, Basly M, Sbai N, et al. Lower urinary trauma complicating gyneacologic and obstetrical surgery. Tunis Med. 2008;86:740-4

24. Giberti C, Germinale F, Lillo M, Bottino P, Simonato A, Carmignani G. Obstetric and gynaecological ureteric injuries: treatment and results. $\mathrm{Br}$ J Urol. 1996;77:21-6. 